\title{
Ueber die Bestimmung der Wendepunkte einer Curve dritter 0rdnung.
}

\author{
Von A. Clemscil in Göttingen.
}

Im Folgenden will ich einige Endformeln zusammenstellen, welche sich auf das Problem der Wendepunkte einer Curve dritter Ordnung beziehen, oder, was dasselbe ist, auf das Problem, die Gleichung einer solchen Curve in die canonische Form

$$
f=a\left(x^{3}+y^{3}+z^{3}\right)+6 b x y z
$$

zu bringen. Von den beiden Constanten $a$ und $b$ kann eine gleich 1 gesetzt werden; es ist aber besser beide zu behalten. Ist

so ist, in Bezug auf die neuen Variabeln $x, y, z$ der Term $6 x y z$ die Function $\left.\Delta_{\chi}^{*}\right)$; sie soll, wie alle in Bezug auf die neuen Variabeln $x, y, z$ ausgeführten Bildungen durch einen obern Strich von der entsprechenden Bildung in Bezug auf die ursprïnglichen Variabeln unterschieden werden. Dann ist also

und also

$$
\Delta_{\%}^{\prime}=6 x y z
$$

daher auch, wenn $r$ die Determinante der Substitution ist:

$$
r^{2} \Delta_{f}=\Delta_{f}^{\prime}=\frac{1}{4}\left(\frac{\partial \Gamma}{\partial a} \Delta_{\chi}^{\prime}-\frac{\partial \Gamma}{\partial b} \chi\right) \text {. }
$$

Die Function $\Gamma$ entsteht aus der Aronhold'schen Function $G$, wenn man die Function $\chi=x^{3}+y^{3}+z^{3}$ an Stelle von $f$ zu Grunde legt: es ist also für diese Form, $\chi$ die erste Invariante gleich Null, die zweite gleich -1 , und demnach

$$
\Gamma=a^{4}+8 a b^{3} \text {. }
$$

Man erhält ferner nach Aronhold folgende Bildungen für die Invarianten:

$$
\left\{\begin{array}{l}
r^{4} S=S^{\prime}=-\frac{1}{144}\left\{\frac{\partial^{\prime} \Gamma}{\partial a^{2}} \frac{\partial^{2} \Gamma}{\partial b^{2}}-\left(\frac{\partial^{2} \Gamma}{\partial a} \partial\right)^{2}\right\}=4 b\left(b^{3}-a^{3}\right) \\
r^{6} T=T^{\prime}=\frac{1}{16}\left\{\frac{\partial \Gamma}{\partial a} \frac{\partial S^{\prime}}{\partial b}-\frac{\partial \Gamma}{\partial b} \frac{\partial S^{\prime}}{\partial a}\right\}=8 b^{6}+20 a^{3} b^{3}-a^{6}
\end{array}\right.
$$

Der Quotient $\frac{b^{3}}{a^{3}}$ bestimmt sich also aus der Gleichung:

*) Die den verschiedenen Invarianten und Covarianten vorzusetzenden numerischen Factoren sind immer wie bei A ro nhold gewählt. (Crelle's Journ. Bd. 55.) 


$$
S^{3}=\frac{64 b^{3}\left(b^{3}-a^{3}\right)^{3}}{\left(8 b^{6}+20 a^{3} b^{4}-a^{6}\right)^{2}}
$$

und zugleich findet man, weun eine der Grössen $a, b$ beliebig angenommen ist, und also $a$ und $b$ mittelst der vorstehenden Gleichung bestimmt sind, die Transformationsdeterminante aus der Gleichung:

$$
r^{2}=\frac{S}{T} \frac{8 b^{6}+20 a^{3} b^{3}-a^{6}}{4 b\left(b^{4}-a^{3}\right)}
$$

Um nun die zu einer Lösung $a, b$ gehörigen $x, y, z$ zu finden, hat man zunïchst aus (4), (5):

$$
\left\{\begin{array}{r}
x^{3}+y^{3}+z^{3}=x=\frac{1}{\Gamma}\left\{\frac{1}{4} f \cdot \frac{\partial \Gamma}{\partial a}-r^{2} \Delta \cdot b\right\} \\
x y z=\frac{1}{6} \Delta_{x}^{\prime}=\frac{1}{6 \Gamma}\left\{\frac{1}{4} f \cdot \frac{\partial r}{\partial b}+r^{2} \Delta \cdot a\right\} .
\end{array}\right.
$$

Benutzen wir ferner die Covariante $\psi$, wie sie Bd. I. p. 57. dieser Annalen definirt ist. Diese Form ist eine Combinante, daher

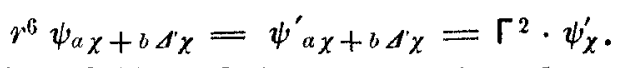

Um aber $\psi_{x}^{\prime}$ zu bilden, hat man aus einer der angeführten Formeln, indem für $\chi S=0, T=-1$ ist:

und

$$
\psi_{\chi}^{\prime}=-3 \varphi_{\chi}^{\prime}+2 \chi^{2}
$$

$$
\varphi_{\chi}^{\prime}=-2\left|\begin{array}{cccc}
x & 0 & 0 & 2 y z \\
0 & y & 0 & 2 z x \\
0 & 0 & z & 2 x y \\
2 y z & 2 z x & 2 x y & 0
\end{array}\right|=8\left(y^{3} z^{3}+z^{3} x^{3}+x^{3} y^{3}\right)
$$

und

$$
\psi_{\chi}^{\prime}=2\left(x^{3}+y^{3}+z^{3}\right)^{2}-24\left(y^{3} z^{3}+z^{3} x^{3}+x^{3} y^{3}\right),
$$

(11) $r^{6} \psi=\Gamma^{2} \cdot\left\{2\left(x^{3}+y^{3}+z^{3}\right)^{2}-24\left(y^{3} z^{3}+z^{3} x^{3}+x^{3} y^{3}\right)\right\}$.

Dies in Verbindung mit (10) giebt folgende weitere Bestimmungen symmetrischer Functionen von $x y z$ :

$$
\left\{\begin{aligned}
y^{3} z^{3}+z^{3} x^{3}+x^{3} y^{3} & =-\frac{r^{6} \psi}{24 \Gamma^{2}}+\frac{1}{12 \Gamma^{2}}\left\{\frac{1}{4} f \cdot \frac{\partial \Gamma}{\partial a}-r^{2} \Delta \cdot b\right\}^{2} \\
x^{6}+y^{6}+z^{6} & =\frac{r^{6} \psi}{12 \Gamma^{2}}+\frac{5}{6 \Gamma^{2}}\left\{\frac{1}{4} f \cdot \frac{\partial \Gamma}{\partial a}-r^{2} \Delta \cdot b\right\}^{2}
\end{aligned}\right.
$$

Ausser diesen symmetrischen Functionen findet sich noch das Differenzenproduct der Grössen $x^{3}, y^{3}, z^{3}$ aus der Function $\Omega$ (a. a. 0 .), welche ebenfalls Combinante ist, und also durch die Formel gegeben wird :

so dass

$$
\begin{aligned}
r^{7} \Omega & =\Omega^{\prime}=\Gamma^{3} \cdot \Omega_{\chi}^{\prime}=144 \Gamma^{3} \cdot\left|\begin{array}{ccc}
x^{5} & x^{2} & y z \\
y^{5} & y^{2} & z x \\
z^{5} & z^{2} & x y
\end{array}\right| \\
& =-144 \Gamma^{3} \cdot\left(x^{3}-y^{3}\right)\left(y^{3}-z^{3}\right)\left(z^{3}-x^{3}\right),
\end{aligned}
$$




$$
\left(x^{3}-y^{3}\right)\left(y^{3}-z^{3}\right)\left(z^{3}-x^{3}\right)=-\frac{r^{9} \Omega}{144 \Gamma^{3}} .
$$

Za bemerken ist, dass das Vorzeichen der rechten Seite nicht bestimmt ist, weil nur $r^{2}$, nicht aber $r$ selbst oben gefunden wurde. Der Ausdruck (13) enthält also aus $\frac{b}{a}$ noch den irrationalen Factor

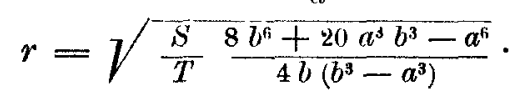

Der numerische Werth der Grösse unter dem Wurzelzeichen besteht aus einer völlig gegebenen, nur von $\left(\frac{b}{a}\right)^{3}$ abhïngigen Grösse, und aus einem willkürlich zu wählenden Factor $b^{2}$, welcher, wie man sieht, aus der Irrationalität als solcher ausscheidet.

Man kann nun mit Hülfe der obigen Data die Cuben und das Product der beiden irrationalen Covarianten

$$
\begin{aligned}
& M=x^{3}+\varepsilon y^{3}+\varepsilon^{2} z^{3} \\
& N=x^{3}+\varepsilon^{2} y^{3}+\varepsilon z^{3}
\end{aligned}
$$

darstellen. Man findet sofort:

$$
\left\{\begin{aligned}
& 8 \Gamma^{3}\left(M^{3}+N^{3}\right)=3 r^{2} \psi\left(\frac{1}{4} f \frac{\partial \Gamma}{\partial a}-r^{2} \Delta b\right)+ \\
&+10\left(\frac{1}{4} f \frac{\partial \Gamma}{\partial a}-r^{2} \Delta b\right)^{3}+216\left(\frac{1}{4} f \frac{\partial \Gamma}{\partial \bar{b}}+r^{2} \Delta a\right)^{3} \\
& \Gamma^{3}\left(M^{3}-N^{3}\right)=3 \varepsilon(\varepsilon-1) r^{9} \Omega \\
& \Gamma^{2} M N=\frac{\psi r^{3}}{8}+\frac{3}{4}\left(\frac{1}{4} f^{\partial} \partial \bar{a}-r^{2} \Delta b\right)^{2} .
\end{aligned}\right.
$$

Man sieht aus den ersten beiden Gleichungen, dass folgende beiden Covarianten neunter Ordnung vollstïndige Cuben sind:

$$
\begin{aligned}
16 \Gamma^{3} M^{3} & =3 r^{2} \psi\left(\frac{1}{4} f \frac{\partial \Gamma}{\partial a}-r^{2} \Delta b\right)+10\left(\frac{1}{4} f \frac{\partial \Gamma}{\partial a}-r^{2} b\right)^{3}+ \\
& +216\left(\frac{1}{4} f \frac{\partial \Gamma}{\partial b}+r^{2} \Delta a\right)^{3}+24 \varepsilon(\varepsilon-1) r^{9} \Omega . \\
16 \Gamma^{3} N^{3} & =3 r^{2} \psi\left(\frac{1}{4} f \frac{\partial \Gamma}{\partial a}-r^{2} \Delta b\right)+10\left(\frac{1}{4} f \frac{\partial \Gamma}{\partial a}-r^{2} b\right)^{3} \\
& +216\left(\frac{1}{4} f \frac{\partial \Gamma}{\partial b}+r^{2} \Delta a\right)^{3}-24 \varepsilon(\varepsilon-1) r^{9} \Omega .
\end{aligned}
$$

Zieht man die Cubikwurzeln, und zwar so, dass der dritten Gleichung (15) genügt wird, so ist schliesslich

$$
\begin{aligned}
& 3 x^{3}=\frac{1}{\Gamma}\left(\frac{1}{4} f \frac{\partial r}{\partial a}-r^{2} \Delta b\right)+M+N \\
& 3 y^{3}=\frac{1}{\Gamma}\left(\frac{1}{4} f \frac{\partial r}{\partial a}-r^{2} \Delta b\right)+\varepsilon M+\varepsilon^{2} N \\
& 3 z^{3}=\frac{1}{\Gamma}\left(\frac{1}{4} f \frac{\partial r}{\partial a}-r^{2} \Delta b\right)+\varepsilon^{2} M+\varepsilon N,
\end{aligned}
$$

wodurch die letzten Darstellungen völlig gegeben sind.

Göttingen, den 20. August 1869. 